\title{
Multifractal Background Noise of Monofractal Signals
}

\author{
D. Grech and G. PamuŁa
}

\author{
Institute of Theoretical Physics, University of Wrocław, PL-50-204 Wrocław, Poland
}

\begin{abstract}
We investigate the presence of multifractal residual background effect for monofractal signals which appears due to the finite length of the signals and (or) due to the constant long memory the signals reveal. This phenomenon is investigated numerically within the multifractal detrended fluctuation analysis (MF-DFA) for artificially generated time series. Next, the analytical formulas enabling to describe the multifractal content in such signals are provided. Final results are shown in the frequently used generalized Hurst exponent $h(q)$ multifractal scenario as a function of time series length $L$ and the autocorrelation scaling exponent value $\gamma$. The obtained results may be significant in any practical application of multifractality, including financial data analysis, because the "true" multifractal effect should be clearly separated from the so called "multifractal noise" resulting from the finite data length. Examples from finance in this context are given. The provided formulas may help to decide whether one deals with the signal of real multifractal origin or not and make further step in analysis of the so called spurious or corrupted multifractality discussed in literature.
\end{abstract}

PACS: 05.45.Tp, 89.75.Da, 05.40.-a, 89.75.-k, 89.65.Gh

Multifractality [1-7] is the property of complex and composite systems that has been attracting more and more attention in recent years in many areas (see e.g. [8-14]). The practical fruits of multifractality are not precisely known yet but in some fields including finance interesting features of this phenomenon were shown (see e.g. [15-24]) that rise hopes for future applications. Since the paper by Kantelhardt et.al. [25] we know that multifractality may result not only from long-range correlations but also from fat tails in probability distributions (PDF) of investigated data even if no memory in data is included $[26,27]$. Normally, one expects multifractality in time series as a result of different autocorrelations appearing at various time scales. This kind of multifractality is most interesting from the practical point of view. However, we always deal in practise with finite samples of data, collected in time series of given length. In such a case multifractality may appear even if no difference in autocorrelation properties exists for various time scales. It is because large fluctuations cannot be detected in finite samples of data with long memory as frequent as small fluctuations - mainly due to the insufficient data statistics. In other words, large fluctuations are not able to be formed in small samples of data, contrary to small fluctuations. Therefore, one gets in the case of shorter time series the apparent multifractal property which itself is not programmed to be multifractal in a sense of different autocorrelation properties at various scales. The latter multifractality, related to variety of autocorrelations, is more substantial and has to be somehow separated from the former one, which we shall call "the multifractal background" or "residual noise" further on.

This residual finite size effects should be distinguished also from the so called spurious and corrupted multifractality $[28,29]$, where the given data series are contaminated by various effects like noise, short-term memory, periodicity in signal, etc., changing the shape of multi- fractal profile. The latter effects will affect multifractality for all lengths of data $L$, while finite size effects disappear if $L \rightarrow \infty$.

The preliminary analysis of this problem had already been made (see e.g. [26-29]). Our goal is to describe the expected level of multifractal background existing due to finite-size effects quantitatively, in terms of generalized Hurst exponents [25] for time series with and without memory, induced by the explicit form of autocorrelations in data. Our approach is based on Fourier filtering method (FFM) [30], directly shaping the artificial data with autocorrelation exponent $\gamma$.

We use the multifractal detrended fluctuation analysis (MF-DFA) [4, 25] as the commonly accepted technique to find multifractal properties of time series. This method is described elsewhere (see e.g. [4, 23-25]) so we will not recall it in details here. To keep the standard notation we will note the $q$-th moment of fluctuation (called also $q$-deformed fluctuation) of the time series signal around its local trend (assumed linear in our approach) in a time window of size $\tau$ for arbitrary $q \in R$ as $F_{q}(\tau)$. Usually, the multifractal properties are presented in the Hölder language as the multifractal spectrum $f(\alpha)$ [7]. Equivalently, in the Hurst language, one can consider the spread of generalized Hurst exponents $h(q)$ [25], calculated for fluctuations $F_{q}(\tau)$ within MF-DFA from the power law:

$$
F_{q}(\tau) \sim \tau^{h(q)}
$$

Both descriptions are linked together via relations [31, 32]

$$
\alpha=h(q)+q h^{\prime}(q), \quad f(\alpha)=q(\alpha-h(q))+1
$$

We will deal in this article only with the generalized Hurst exponent description of multifractality. Nevertheless, our results can be easy translated into Hölder language with the use of Eq.(2).

Our aim is to evaluate the multifractal effect of finite artificial signals of various lengths for the given constant persistency level, i.e. persistency not changing with the 
time scale. Such signals are built by us within FFM [30]. The level of autocorrelations is directly modulated by the proper choice of scaling exponent $\gamma$ responsible for the magnitude of autocorrelation function $C(\tau)$. The autocorrelation function $C(\tau)$ satisfies for stationary series with long memory the known power law:

$$
C(\tau) \simeq\langle\Delta x(t) \Delta x(t+\tau)\rangle \sim \tau^{-\gamma}
$$

where $\Delta x(t)=x(t+1)-x(t)$ are increments of discrete time series, $\tau$ is the time-lag between observations and the average \langle\rangle is taken over all data in series.

The $\gamma$ scaling exponent may be linked to the Hurst exponent $H$ [33] by the formula [34]:

$$
\gamma=2-2 H
$$

In the quantitative analysis of residual multifractality left in monofractal finite signals, we concerned the ensembles of numerically generated time series of length $L=2^{n}$, $(n=9,10, \ldots, 20)$ with the pre-assumed autocorrelation exponent value $\gamma=0.1,0.2, \ldots, 0.9,1.0$, each containing $10^{2}$ independent realizations. Thus, the spread of $\gamma$ exponents covers the range $1 / 2 \leq H<1$. The every obtained quantity has been averaged over such statistical ensemble produced for the $L$ and $\gamma$ values as input parameters.

First, we examined the FFM procedure for time series generation, in order to check its accuracy towards replication of the pre-assumed autocorrelation properties coming out from the particular choice of $\gamma$ exponent as input. Fig. 1 demonstrates its efficiency. It is seen that the power law in Eq.(3) is very well reproduced. Moreover, a coincidence between input and output $\gamma$ 's is also satisfactory. The length of generated data-samples was chosen as powers of 2 to improve performance of the fast Fourier transform algorithm.

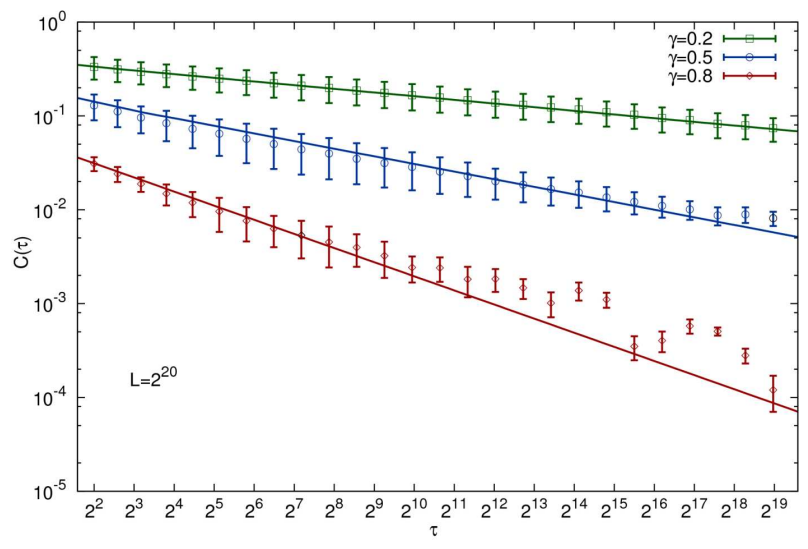

Fig. 1. Efficiency of FFM for replication of autocorrelation properties in time series. The examples for input values $\gamma=0.2,0.5,0.8$ are shown in $\log$-log scale for the generated data of length $L=2^{20}$. The solid lines present the fit to the desired power law dependence of Eq.(3), while error-bars show $1 \sigma$ standard deviation following from the considered statistics of $10^{2}$ independent realizations. The output $\gamma$ values from the fit are found $\gamma_{\text {out }}=0.203( \pm 0.009), 0.498( \pm 0.012), 0.782( \pm 0.054)$ respectively.
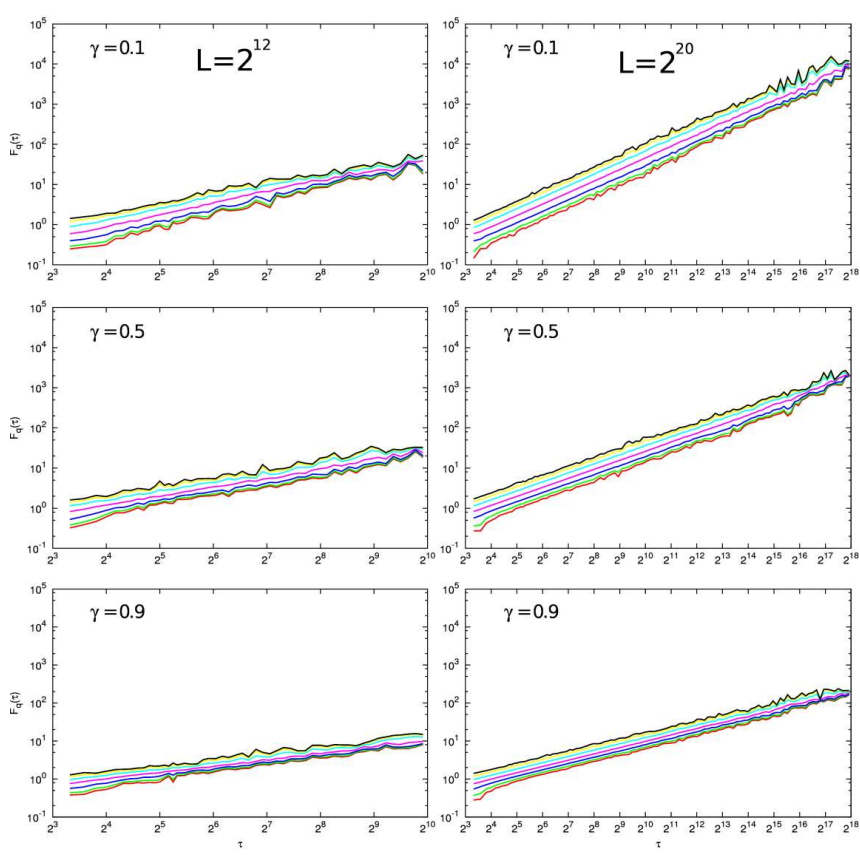

Fig. 2. Scaling properties of $q$-deformed fluctuations within MF-DFA. Results are presented for two different lengths of time series $L=2^{12}, 2^{20}$, three autocorrelation parameters $\gamma=0.1,0.5,0.9$ and $q=$ $-15,-10,-5,0,+5,+10,+15$ (from bottom to top). All plots confirm the proposed scaling range from $\tau=10$ till $\tau=L / 4$.

The next problem we had to examine was the performance of MF-DFA technique which strictly depends on the power law scaling between $q$ - deformed fluctuations $F_{q}(\tau)$ and the window size $\tau$ (see Eq. $\left.(1)\right)$. An exact extraction of the generalized Hurst exponent $h(q)$ is then possible only for well determined scaling range in the fitting procedure $\log F_{q}(\tau)$ vs $\log \tau$. Fig. 2 clearly shows the expected power law dependence for various lengths $L$ of the signal and for different values of deformation parameter $q$ uniformly distributed in the range from -15 to +15 . These plots justify the scaling range from $\tau=10$ till $\tau=L / 4$, chosen by us further on.

To determine quantitatively the amount of multifractal residual noise present in given time series, the edge values of $h(q)$ function were investigated for them. Let us introduce the new parameter $\Delta h$ defined as the difference between the two asymptotic limits:

$$
\Delta h=\lim _{q \rightarrow-\infty} h(q)-\lim _{q \rightarrow \infty} h(q)
$$

and assume for numerical reasons that such asymptotic limits are reached already at $q= \pm 15$. Such assumption is justified in Fig. 3, where plots for $h(q)$ are shown for $L=2^{12}, 2^{20}$ and for $\gamma=0.1,0.5,, 0.9$ respectively.

Generally, we may expect that $\Delta h$ is the unknown function of $L$ and $\gamma$. The form of $\Delta h(L, \gamma)$ dependence is thus a crucial problem. To simplify it, one may consider the case $\gamma=$ const for a moment, e.g. $\gamma=1(H=1 / 2)$ corresponding to uncorrelated data. Fig. 4 shows the 

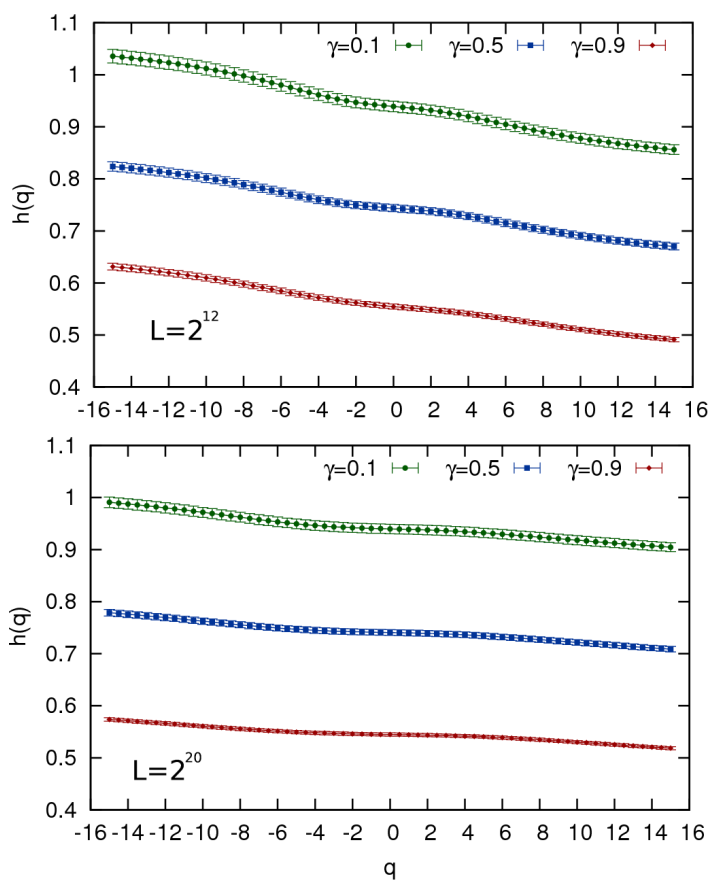

Fig. 3. Generalized Hurst exponent for monofractal signals generated within FFM with various autocorrelation properties. Two cases, for $L=2^{12}$ and $L=2^{20}$ are shown with different autocorrelation levels. Error bars correspond to statistics $(1 \sigma)$ of $10^{2}$ generated series.

edge characteristics of $h(q)$ calculated for two distinct series of length $L=2^{12}, 2^{20}$, generated with the autocorrelation exponent $\gamma$ and then shuffled. The dependence on $\gamma$ is evidently absent proving that shuffling procedure was effective enough, while the dependence $\Delta h( \pm 15)$ on $L$ is still kept and obvious.

The detailed analysis of the latter relationship is revealed in Fig. 5 collecting results for various data lengths. Astonishingly, this figure suggests a power law dependence between $\Delta h_{1} \equiv \Delta h(\gamma=1)$ and $L$

$$
\Delta h_{1}(L)=C_{1} L^{-\eta_{1}}
$$

where $C_{1}$ and $\eta_{1}$ are constant.

The knowledge of $95 \%$ confidence level for this relation is crucial in practise. This level means that any result measured above the particular value has probability less than $5 \%$. To obtain this confidence level one has to correct $C_{1}$ and $\eta_{1}$ parameters by the corresponding quantiles calculated from the $1 \sigma$ uncertainties $\sigma_{C_{1}}, \sigma_{\eta_{1}}$ of the fit and from the standard deviation $S_{1}$ resulting from the series statistics*:

$$
\Delta h_{1}^{95 \%}(L)=C_{1} \exp \left(f\left(\sigma_{C_{1}}+S_{1}\right)\right) L^{-\eta_{1}+f \sigma_{\eta_{1}}}
$$

where $f=1.65$ is the respective factor for the particular $95 \%$ confidence level.

\footnotetext{
* exponential dependence in this formula comes from the uncertainty of regression line fit in logarithmic scale
}
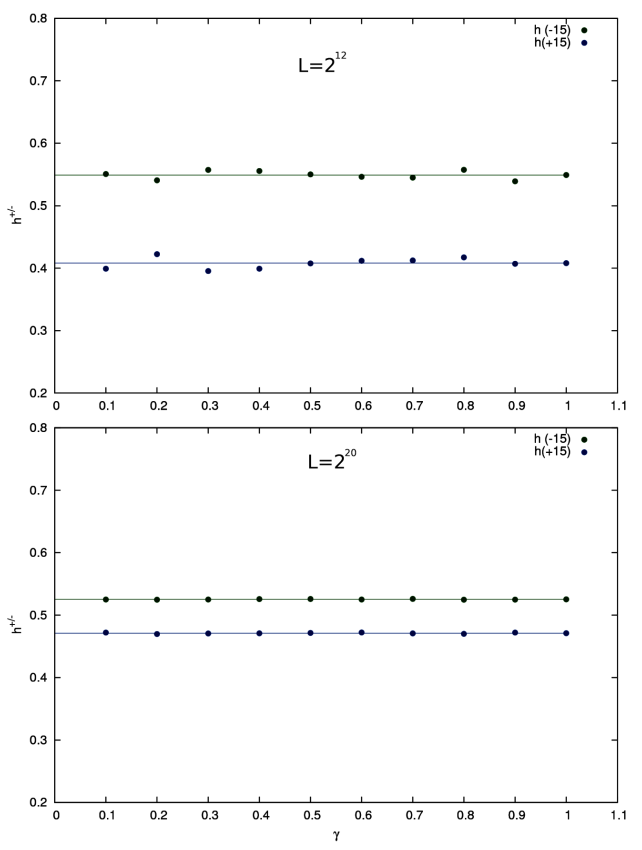

Fig. 4. Edge values of the generalized Hurst exponents $h(q)$ for two different lengths of time series $L=2^{12}, 2^{20}$ constructed with long memory present $(\gamma<1)$ and then shuffled to kill this memory. Dependence on the data length is readable.

Let us take now a closer look at the case of autocorrelated $(0 \leq \gamma<1)$ finite signals. The edge values for $h( \pm 15)$ versus the autocorrelation exponent value $\gamma$ were investigated again, keeping $L$ fixed. The examples of this dependence for $L=2^{12}$ and $L=2^{20}$ are shown in Fig. 6. We found that cases for other lengths (not shown) look similarly and indicate the excellent linear, decreasing function of $h( \pm 15)$ versus $\gamma$ in the whole range of autocorrelation exponent. Thus one gets:

$$
\Delta h(\gamma, L)=A(L) \gamma+B(L)
$$

where the linear coefficients $A(L)$ and $B(L)$ depend on $L$ only. They can be further specified with the use of boundary relations, i.e. the form of $\Delta h_{1}(L)$ and $\Delta h_{0}(L) \equiv \Delta h(\gamma=0, L)$ functions.

The first boundary condition, i.e. $\Delta h_{1}(L)$, was already specified in Eq.(6). The profile of the second one $\left(\Delta h_{0}(L)\right)$ can be deduced from Figs.6,7. The extrapolation of the fitting lines $h( \pm 15)$ versus $\gamma$ to the point $\gamma \rightarrow 0$ is needed because the case $\gamma=0$ is not accessible directly in FFM (see Fig. 6). Their results give the collection of $\Delta h(0, L)$ values, plotted against the length of time series in Fig. 7. This can be done for the central values as well as for the data satisfying $95 \%$ confidence level. It is seen from Fig. 7 that for fully autocorrelated time series $(\gamma \rightarrow 0) \Delta h_{0}(L)$ is represented again by the power law:

$$
\Delta h(0, L)=C_{0} L^{-\eta_{0}}
$$

with some constants $C_{0}$ and $\eta_{0}$ to be determined from the fit. 


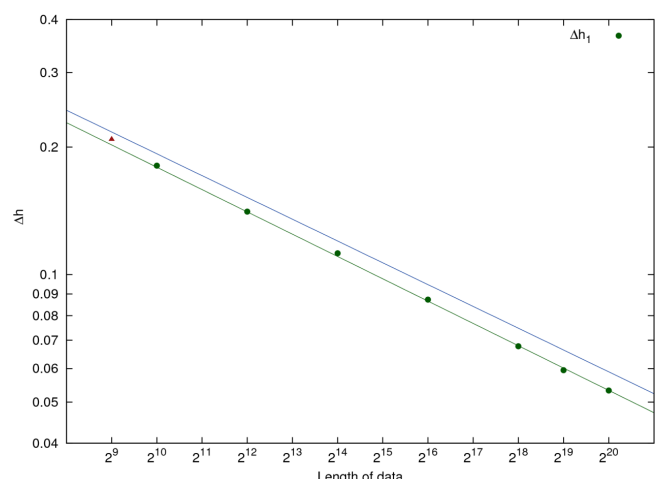

Fig. 5. Spread $\Delta h_{1}$ of generalized Hurst exponent versus length of data drawn in log-log scale for the signal with no memory. Power-law dependence between $\Delta h$ and the data length is visible. Data point corresponding to $L=2^{9}$ (marked as triangle) is slightly above the fitting line due to insufficient statistics for short signal. Therefore, this point has been neglected during fitting procedure. Results of the fit are $C_{1}=0.603, \eta_{1}=0.175$ for the central values and $C_{1}^{95 \%}=0.631, \eta_{1}^{95 \%}=0.171$ for $95 \%$ confidence level. The latter one is marked as the top line..

Linking the shape of boundary conditions given in Eqs.(6) and (9) with the general linear dependence in Eq.(8), one arrives with the final formula for $\Delta h(\gamma, L)$ :

$$
\Delta h(\gamma, L)=C_{1} L^{-\eta_{1}} \gamma+C_{0} L^{-\eta_{0}}(1-\gamma)
$$

The shape of the $95 \%$ confidence level for multifractal background noise will be given by the same formula but with different coefficients calculated as in Eq.(7). The final values of these coefficients are collected in Table I, with uncertainties presented in Table 2II

Our results may also be presented graphically in a form of 'phase-like' diagrams (see Fig. 8). Three separable areas in $(\Delta h, \gamma)$ plane can be distinguished for every $L$. The first area corresponds to multifractality connected entirely with finite size effects and is not related to autocorrelations at all. It is marked in red in Fig. 8. The second domain, marked in light green, is related to $(\Delta h, \gamma)$ range, where the apparent multifractality manifests as a result of finite length of data with the constant (i.e independent on the chosen time scale) level of long memory. The 'true' multifractality, i.e. related with long memory entirely dependent on the time scale, may occur only in the white region (at 95\% confidence level).

Finally, one should compare the obtained multifractal noise thresholds with examples of the real multifractal data. We took them from finance because of common agreement that multifractality is a characteristic feature of financial markets. This problem is considered in Fig. 9, where the simulated 'phase-like' diagram for data length $L \sim 2 \times 10^{3}$ is shown, together with multifractal properties of various markets. The particular length $L$ has been chosen as the average length of available data for analysed stocks. The multifractal properties for price indices were taken from Ref. [35].

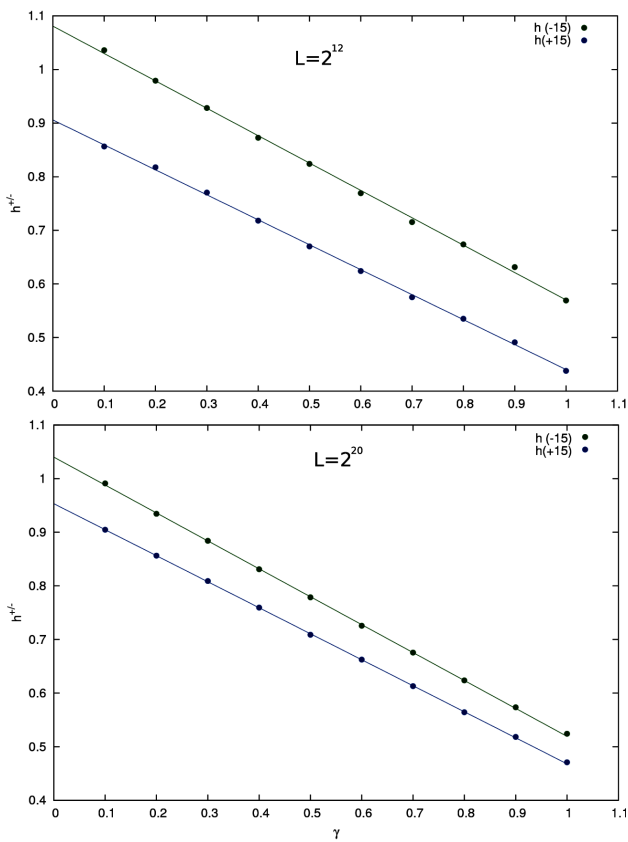

Fig. 6. Edge values of generalized Hurst exponent for series with long-memory. Figures clearly show the linear dependence between the edge values $h^{ \pm} \equiv h( \pm 15)$ and $\gamma$ exponent. Extrapolation of fitted lines to the point $\gamma=0$ is interpreted as the edge values for fully autocorrelated signal $(C(\tau) \rightarrow 1, \forall \tau)$.

It is seen that the presence of multifractality for the price index data is not so evident for all markets. One may find indices where it comes indeed as a result of scaling properties changing with the time scale (e.g. Venezuela, Indonesia, China). Simultaneously, there are markets where the observed multifractality is generated mainly (e.g. Philippines, Taiwan, Germany) or even entirely (Ireland) by the finite size effects. In the case of Philippines, Taiwan, Thailand, Germany, Spain, Greece, almost $80 \%$ of the apparent multifractal behavior in price indices is related to such an effect. The case of Ireland is even more intriguing because the observed multifractality is the false signal coming entirely from finite size effects.

Concluding, we have shown quantitatively how multifractality arises from the finite size effects and (or) from autocorrelations not changing with the time scale and formed by the specific $\gamma$ autocorrelation exponent. This kind of multifractality, called by us "multifractal noise", should be clearly distinguished from the real multifractality caused by memory effects dependent on the time scale and thus leading to different scaling properties at various scales. These quantitative findings confirm and push further the study of the generalized Hurst exponent spread suggested in [29] as not indicative for multifractality if $\Delta h \leq 0.2$. Our approach differs from that in Ref. [26] because we used direct autocorrelation input between data (see Eq.(3)). The provided analytical formulas de- 


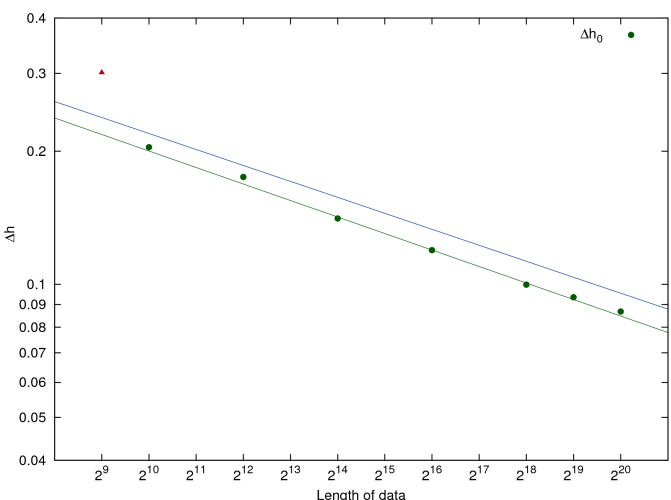

Fig. 7. Spread $\Delta h_{0}$ of generalized Hurst exponent for fully autocorrelated time series $(\gamma=0)$ versus the length of data. Solid green line presents the power-law fit in $\log$-log scale and the top blue line corresponds to $95 \%$ confidence level resulting from fit uncertainty and statistics. Fitted parameters are $C_{0}=0.453, \eta_{0}=0.124$ and $C_{0}^{95 \%}=0.484, \eta_{0}^{95 \%}=0.120$. Data point corresponding to $L=2^{9}$ has been removed from the fit due to insufficient statistics for so short signal leading to huge uncertainty in the estimation of generalized Hurst exponents within MF-DFA.

TABLE I

Results of the best fit for coefficients in Eq.(10) and for their $95 \%$ confidence level describing the multifractal threshold caused by the finite size effects.

\begin{tabular}{c|c|c|c||c|c|c|c}
\hline \hline$C_{1}$ & $\eta_{1}$ & $C_{0}$ & $\eta_{0}$ & $C_{1}^{95 \%}$ & $\eta_{1}^{95 \%}$ & $C_{0}^{95 \%}$ & $\eta_{0}^{95 \%}$ \\
\hline 0.603 & 0.175 & 0.453 & 0.124 & 0.631 & 0.171 & 0.484 & 0.120
\end{tabular}

scribe the multifractal noise threshold as the power law function of time series length and the linear function of autocorrelation exponent $\gamma$. It has been shown that in the case of real financial data, their multifractal properties may substantially (even in $80 \%-100 \%$ ) originate from the multifractal noise caused by finite size effects. It makes difficult in some cases to separate what main phenomenon is really responsible for the effect one observes. The presence of changing trends, periodicity and additive noise described for artificial data in [28], and dis-

\section{TABLE II}

Uncertainty of the fit and statistical uncertainty $\left(S_{0}, S_{1}\right)$ at $1 \sigma$ level rounded up to $10^{-3}$ for coefficients in Eq.(10) describing the multifractal noise thresholds. Subscripts correspond to $\gamma=0$ and $\gamma=1$ cases respectively.

\begin{tabular}{c|c|c||c|c|c}
\hline \hline$\sigma_{C_{1}}$ & $\sigma_{\eta_{1}}$ & $S_{1}$ & $\sigma_{C_{0}}$ & $\sigma_{\eta_{0}}$ & $S_{0}$ \\
\hline 0.006 & 0.003 & 0.034 & 0.008 & 0.003 & 0.032
\end{tabular}
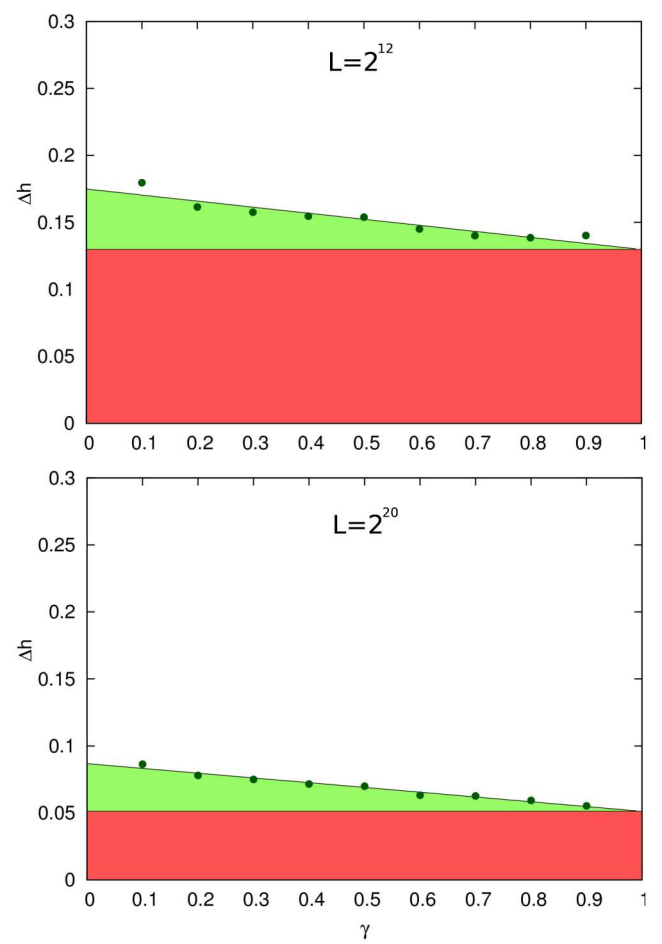

Fig. 8. Examples of phase-like diagrams for two data lengths $L=2^{12}$ and $L=2^{20}$. Three separable areas can be distinguished, marked in red, light green and white respectively. They are related to $(\Delta h, \gamma)$ domains where multifractality is caused at $95 \%$ confidence level correspondingly: by finite size effects, constant autocorrelation level independent on the time scale and 'true' multifractality (white).

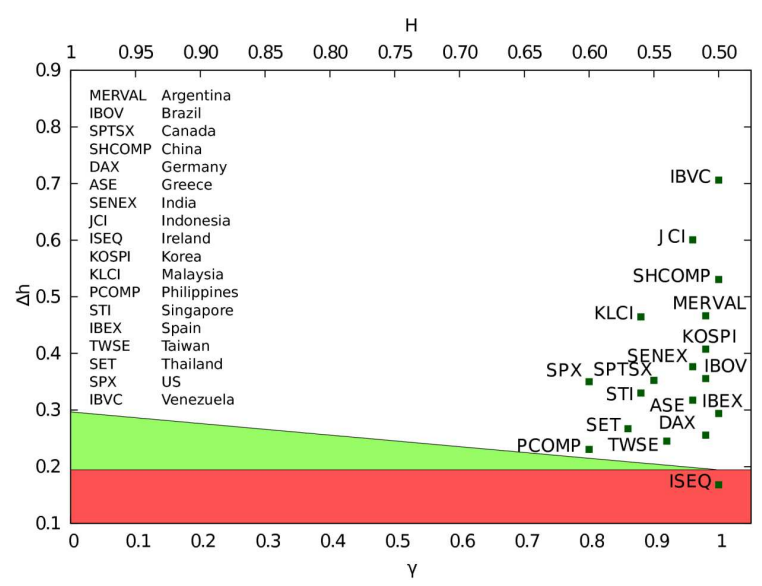

Fig. 9. Simulated 'phase-like' diagram for $L=2 \times 10^{3}$ with outlined market data. Squares represent multifractal properties of price indices and data were taken from Ref.[35]. The Bloomberg code has been used to describe markets. The corresponding values for Hurst exponents $H=h(2)$ are also indicated (top axis) for convenience.. 
cussed in some extend earlier, in the framework of abrupt events for real data in [24], makes this analysis even more complicated.

This confirms that multifractality is very tiny and delicate effect and one should be especially careful drawing far-reaching conclusions from the multifractal analysis. Our formulas are general enough to be applied also to real data in other areas, in order to distinguish if and how their multifractal properties have real multifractal origin.

\section{References}

[1] S. Ghashghaie, W. Breymann, J. Peinke, P. Talkner, Y. Dodge, Nature 381, 767 (1996).

[2] R.N. Mantegna, H.E. Stanley, Nature 383, 587 (1996).

[3] B.B. Mandelbrot, Sci. Am. 298, 70 (1999).

[4] J.W. Kantelhardt, arXiv: 0804.0747v1 [phys.dataan].

[5] Z. Eisler, J. Kertész, Physica A 343, 603 (2004).

[6] H.G.E. Hentschel, I. Procaccia, Physica D 8, 435 (1983).

[7] T.C Halsey, M.H. Jensen, L.P. Kadanoff, I. Procaccia, B.I. Shraiman, Phys. Rev. A 33, 1141 (1983).

[8] J.F. Muzy, E. Bacry, A. Arneodo, Phys. Rev. Lett. 67, 3515 (1991).

[9] P.P. Dimitriu, E.M. Scordilis, V.G. Karacostas, Natural Hazards 21, 277 (2000); J.W. Kantelhardt, D. Rybski, S.A. Zschiegner, P. Braun, E. KoscielnyBunde, V. Livina, S. Havlin, A. Bunde, Physica A 330, 240 (2003); Y. Ashkenazy, D.R. Baker, H. Gildor, S. Havlin, Geophys. Res. Lett. 30, 2146 (2003); R.G. Kavasseri, R. Nagarajan, Chaos, Solitons, Fractals. 24, 165 (2005).

[10] L.F. Burlaga, J. Geophys. Res. 97, 4283 (1992); Z. Vőroôs, W. Baumjohann, R. Nakamura, A. Runov, T.L. Zhang, M. Volwerk, H.U. Eichelberger, A. Balogh, T.S. Horbury, K.H. Glassmeier, B. Klecker, H. Reme, Ann. Geophys. 21, 1955 (2003).

[11] D.W. Chappell, J. Scalo, Astrophys. J. 551, 712 (2001); V.I. Abramenko, Solar Phys. 228, 29 (2005); M.S. Movahed, G.R. Jafari, F. Ghasemi, S. Rahvar, M.R.R. Tabar, J. Stat. Mech. P02003 (2006).

[12] P.Ch. Ivanov, L.A.N. Amaral, A.L. Goldberger, S. Havlin, M.G. Rosenblum, Z.R. Struzik, H.E. Stanley, Nature 399, 461 (1999); B.J. West, M. Latka, M. Glaubic-Latka, D. Latka, Physica A 318, 453 (2003); M.S. Baptista, L.O.B. de Almeida, J.F.W. Slaets, R. Koberle, C. Grebogi, Phil. Trans, R. Soc. A 366, 345 (2008).
[13] D. Makowiec, A. Dudkowska, R. Gałąska, A. Rynkiewicz, Physica A 388, 3486 (2009).

[14] G. Bianconi, A.-L. Barabási, Europhys. Lett. 54, 436 (2001); G. Zhu, H.J. Yang, C.Y. Yin, B.W. Li, Phys. Rev. E 77, 066113 (2008).

[15] K. Matia, Y. Ashkenazy, H.E. Stanley, Europhys. Lett. 61, 422 (2003).

[16] J. Kwapień, P. Oświȩcimka, S. Drożdż, Physica A 350, 466 (2005).

[17] P. Oświęcimka, J. Kwapień, S. Drożdż, Physica A 347, 626 (2005).

[18] L.G. Moyana, J. de Souza, S.M.D. Queiros, Physica A 371, 118 (2006).

[19] J. Jiang, K. Ma, X. Cai, Physica A 378, 399 (2007).

[20] K.E. Lee, J.W. Lee, Physica A 383, 65 (2007).

[21] G. Lim, S. Kim, H. Lee, K. Kim, D.-I. Lee, Physica A 386, 259 (2007)

[22] Z.-Y. Su, Y.-T.Wang, H.-Y. Huang, J. Korean Phys. Soc. 54, 1395 (2009).

[23] P. Oświęcimka, J. Kwapień, S. Drożdż, A.Z. Górski, R. Rak, Acta Phys. Pol. A 114, 3 (2008).

[24] Ł. Czarnecki, D. Grech, Acta Phys. Pol. A 117, 4 (2010).

[25] J.W. Kantelhardt, S.A. Zschiegner, E. KoscielnyBunde, S. Havlin, A. Bunde, H.E. Stanley, Physica A 316, 87 (2002).

[26] Wei-Xing Zhou, arXiv:0912.4782v1 [q-fin.ST].

[27] S. Drożdż, J. Kwapień, P. Oświęcimka, R. Rak, Europhys. Lett. 88, 60003 (2009), arXiv:0907.2866 [physics.data-an].

[28] J. Ludescher, M.I. Bogachev, J.W. Kantelhardt, A.Y Schumann, A. Bunde, Physica A 390, 2480 (2011).

[29] A.Y. Schumann, J.W. Kantelhardt, Physica A 390, 2637 (2011)

[30] H.A. Makse, S. Havlin, M. Schwartz, H.E. Stanley, Phys. Rev. E 53, 2 (1996).

[31] J. Feder, Fractals, New York, Plenum Press (1988).

[32] H.-O. Peitgen, H. Jürgens, D. Saupe, Chaos and Fractals, 2nd ed. Springer, 2004.

[33] H.E. Hurst, Trans. Am. Soc. Civ. Eng. 116, 770 (1951).

[34] J.W. Kantelhardt, E. Koscielny-Bunde, H.H.A. Rego, S. Havlin , A. Bunde, Physica A 295, 441 (2001).

[35] L. Zunino, B.M. Tabak, A.Figliola, D.G. Pérez, M. Garavaglia, O.A. Rosso, Physica A 387, 6558 (2008). 